
allemande

46-2 | 2014

Intellectuels et politique en Allemagne

\title{
La « mise au pas » (Gleichschaltung) de l'Alsace- Moselle en 1940-1942
}

Défrancisation, décléricalisation, germanisation, nazification

Jean-Noël Grandhomme

\section{(2) OpenEdition}

\section{Journals}

Édition électronique

URL : https://journals.openedition.org/allemagne/1844

DOI : $10.4000 /$ allemagne. 1844

ISSN : 2605-7913

Éditeur :

Société d'études allemandes, Presses universitaires de Strasbourg

Édition imprimée

Date de publication : 30 décembre 2014

Pagination : 443-465

ISSN : 0035-0974

Référence électronique

Jean-Noël Grandhomme, "La « mise au pas » (Gleichschaltung) de l'Alsace-Moselle en 1940-1942 », Revue d'Allemagne et des pays de langue allemande [En ligne], 46-2 | 2014, mis en ligne le 29 juillet 2019, consulté le 16 février 2023. URL : http://journals.openedition.org/allemagne/1844 ; DOI : https:// doi.org/10.4000/allemagne.1844 


\section{La « mise au pas » (Gleichschaltung) de l'Alsace-Moselle en 1940-1942 Défrancisation, décléricalisation, germanisation, nazification}

\section{- Jean-Noël Grandhomme*}

Au cours de la Seconde Guerre mondiale, certaines parties de l'Europe, dans un premier temps occupées par la Wehrmacht (entre 1939 et 1941), connaissent ensuite une annexion de fait au $\mathrm{III}^{\mathrm{e}}$ Reich. C'est le cas de plusieurs régions de Pologne, des cantons de l'Est (Eupen et Malmédy) en Belgique, du Luxembourg, du nord de la Slovénie et de l'AlsaceMoselle. Dans ces zones les nazis pratiquent une politique d'assimilation à marche forcée, qui se caractérise partout par une phase initiale de « mise au pas » ou Gleichschaltung.

Ainsi, fermement décidée à ne pas permettre la renaissance du particularisme alsacien-lorrain de la période 1871-1918, l'Allemagne, même si elle annexe de fait le Reichsland dans ses frontières exactes d'autrefois, le disloque aussitôt en rattachant l'Alsace au Gau Baden, rebaptisé Oberrhein (qui correspondait avant-guerre au Pays de Bade), et en incluant (le 30 novembre 1940) la Moselle dans le nouveau Gau Westmark (avec la Sarre et le Palatinat). À la tête de ces deux entités administratives, Hitler installe des fidèles, de vieux compagnons, deux " pédagogues »: l'ancien instituteur Josef Bürckel $^{(1)}$ et l'ancien élève de l'école normale Robert Wagner ${ }^{(2)}$, avec le titre de chef de l'administration civile (Chef der Zivilverwaltung).

* Maître de conférences (HdR) en histoire contemporaine à l'Université de Strasbourg, membre du conseil scientifique du Mémorial d'Alsace-Moselle, membre de l'EA Arche.

1 Né à Lingenfeld (Palatinat) en 1895, il est chargé de la réintégration de la Sarre au Reich, puis nommé Reichsstatthalter (gouverneur) d'Autriche et Gauleiter de Vienne. Envoyé en Lorraine le 2 août 1940, il tombe progressivement en disgrâce en raison de son tempérament indépendant. Accusé de tiédeur dans les mesures de défense prises face à l'avance des Alliés, il est sans doute contraint à un suicide déguisé en accident à Neustadt (Haardt) le 28 septembre 1944. Le 4 octobre, Willi Stöhr le remplace. Né en 1903 à Wuppertal-Elberfeld (Rhénanie du Nord-Westphalie), il n'obtient le titre de Gauleiter que le $1^{\text {er }}$ février 1945 alors que la Lorraine annexée ne se réduit plus qu'à quelques communes. Il aurait encore été identifié au Canada en 1994.

2 Né à Lindach (Bade) en 1895, Wagner a participé au putsch de la Brasserie. Gauleiter du Pays de Bade en 1933, il se voit confier le 2 août 1940 l'administration civile de l'Alsace. Arrêté à la suite 
Wagner comme Bürckel héritent de deux territoires en partie dépeuplés, dont les infrastructures ont souffert des combats de juin 1940. Dans un premier temps - celui de la séduction -, ils s'attachent à faire rentrer au pays les prisonniers de guerre et les évacués (sauf les indésirables, comme les cadres de la fonction publique française) et à remettre en état routes, ponts et voies de chemin de fer. Ensuite, très rapidement, les nouveaux territoires sont alignés sur le Reich. "La région a été mise au pas, vraiment, écrit l'historien allemand Lothar Kettenacker: ce qui s'est passé en Allemagne sur quelques années s'est passé ici en quelques semaines » ${ }^{(3)}$.

Ce sont ces opérations de déracinement (défrancisation), de décléricalisation et d'assimilation (germanisation et nazification) qui constituent ici notre propos. Nous les envisageons à la fois d'une manière chronologique et thématique, en ayant souvent recours aux témoignages, en grande partie inédits ou publiés dans des éditions confidentielles.

\section{La libération des prisonniers de guerre}

Présentée comme une mesure de faveur à l'égard de soldats d'origine allemande (Deutschstämmig erachtete) en quelque sorte enrôlés dans une armée «étrangère » (du point de vue pangermaniste), celle de la France, la libération des prisonniers de guerre constitue l'une des mesures les plus spectaculaires aussitôt décidées par les nazis. Si quelques rares soldats l'ont refusée, la plupart - comme c'est bien compréhensible l'ont acceptée volontiers. Les Allemands extraient rapidement des Stalag et des Oflag les Alsaciens et les Lorrains mosellans qu'ils ont décidé de libérer en signe d'apaisement et d'inauguration sous d'heureux auspices du nouveau régime (encore flou, d'ailleurs) sous lequel sont passées leurs provinces. C. Z., de Montois-la-Montagne (Moselle), capturé dans les Vosges le 23 juin 1940, interné à Strasbourg, est quant à lui renvoyé dans ses foyers en qualité de Lorrain dès le 5 juillet, quelques heures à peine après la reddition des dernières troupes de la Ligne Maginot ${ }^{(4)}$.

Certains prisonniers, comme Louis Borr, capturé à Lille, sont d'autant plus heureux de quitter leur camp que les relations avec les « Français de l’Intérieur » n’y étaient pas toujours bonnes. «Beaucoup d'Alsaciens qui étaient là parlaient patois entre eux. Un sous-lieutenant qui est passé a dit qu'on était des traîtres " ${ }^{(5)}$. La mesure ne touche pas les officiers généraux. Le général Rinck $(1878-1953)^{(6)}$, commandant de l’artillerie du

d'une longue traque, il est fusillé à Strasbourg le 14 août 1946. Voir Ludger Syre, « Der Führer vom Oberrhein: Robert Wagner, Gauleiter, Reichsstatthalter in Baden und Chef der Zivil Verwaltung im Elsaß », in: Michael Kissener, Die Führer des Provinz: NS-Biographien aus Baden und Württemberg, Constance, 1997, p. 733-779; Lothar Kettenacker, « La politique de nazification en Alsace. Première partie: Le témoignage d'un Allemand », Saisons d'Alsace, n 65 (1978), p. 61-75 et Jean-Laurent VonAu, Le Gauleiter Wagner. Le bourreau de l'Alsace, Strasbourg, 2011.

4 Archives départementales de la Moselle (ADM), 1486 W 652, PV de la gendarmerie de Bouzonville, 15 juillet 1959. Dossier personnel. Direction interdépartementale des Anciens combattants LorraineChampagne-Ardenne.

5 Témoignage oral (TO), Louis Borr, Volmerange-lès-Boulay, 6 octobre 2008.

6 Né à Wasserbourg (Bas-Rhin), commandant supérieur des troupes coloniales dans la métropole (19371939). 
corps d'armée colonial au début de la guerre, lui aussi fait prisonnier le 23 juin 1940, n'est libéré que le 12 mai 1945. Cent cinquante officiers restent également dans les camps car ils ont refusé de solliciter leur retour.

Dans une directive du 30 août le général Colson, secrétaire d'État à la Guerre de Vichy, demande aux commandants des régions militaires de "ne pas décourager » les militaires alsaciens-mosellans de rentrer chez eux. Aux soldats qui éprouvent des scrupules à signer un document dans lequel ils se reconnaissent " Alsacien-Lorrain, de race germanique, né en Alsace avant 1918 ", Colson accorde par avance le pardon de la France: les « autorités françaises les considèrent comme déliés de tout engagement que les autorités occupantes pourraient exiger d'eux comme condition de leur rapatriement ». L'État français estime donc opportun de saisir l'occasion offerte par les Allemands, afin de repeupler l'Alsace et la Moselle d'éléments considérés comme parmi les plus fidèles à la France car ils ont porté son uniforme, se sont battus et ont parfois été blessés pour elle. On tient à tout prix à ne pas renouveler l'erreur de 1872 où le droit d'option avait conduit au départ des soutiens de la France parmi les plus actifs, progressivement remplacés par des sujets allemands, ce qui fut considéré, dit-on, par Maurice Barrès, comme « un second Sedan ».

Les nazis ont exclu des mesures de faveur - d'ailleurs partiellement appliquées aussi à des activistes flamingants, bretons ou autres - les israélites, ainsi que ceux qui ont demandé à se retirer en zone libre ou même occupée, ce qui est considéré comme un signe de méfiance si ce n'est d'hostilité ${ }^{(7)}$. Évidemment, sous de fausses couleurs désintéressées, leur politique vise à creuser un fossé entre les Alsaciens-Mosellans et leurs compatriotes des autres départements français, les premiers bénéficiant ostensiblement de privilèges. La manœuvre des nazis est plus habile encore à un autre titre, et les autorités militaires françaises, pour le coup clairvoyantes, entrevoient une conséquence possible. En effet, la signature de la déclaration implique pour eux (sans que la plupart en aient encore le moindre soupçon) des « devoirs envers la "Patrie allemande", c'est-à-dire l'engagement à servir éventuellement dans les armées du III ${ }^{\mathrm{e}}$ eich ${ }^{(8)}$. Les plus jeunes de ces libérés, en effet, seront ensuite incorporés de force dans la Wehrmacht.

\section{Le retour des évacués}

À partir de juillet 1940, environ 500000 des peut-être 630000 évacués et mobilisés alsaciens et lorrains mosellans acceptent de revenir dans leur foyer, encouragés par les Allemands mais surtout saisis par le Heimweh (mal du pays), désireux de revoir leur foyer et de retrouver leur famille ${ }^{(9)}$. Certains jeunes gens n'éprouvent aucun état d'âme à l'idée de ce retour. "Fin 1940, quand ma famille évacuée en Charente est retournée à Rémelfing, je dois reconnaître que je n’avais aucune conscience des événements, écrit François Rothan. Je venais d'avoir quatorze ans, j'avais des frères et

7 Document non daté (automne de 1940) tiré de la collection Thuet, dans André Hugel, Nicolas MenGUs, Entre deux fronts. Les incorporés de force alsaciens dans la Waffen SS, vol. 1, Paris/Sarreguemines, 2007, p. 12.

$8 \quad$ Ibid.

9 La Voix des groupements GERAL-PRAF, juillet 1997, n 319. 
des sœurs, et à part la mécanique à l'école professionnelle, une seule chose me passionnait: le foot » ${ }^{(10)}$. De leur côté, les parents de Jean Amos avaient perdu une bonne partie de leurs biens dans la destruction de la maison d'un parent. "Alors ils étaient naturellement assez déprimés et ils voulaient rentrer à Strasbourg. Que voulez-vous?! En fils obéissant, je suis rentré avec eux, ma sœur aussi », explique, un peu amer, celui pour qui cette décision a eu ensuite pour conséquence son incorporation de force dans l'armée allemande ${ }^{(11)}$. Le poids de l'autorité parentale explique aussi le retour d'Alfred Bapst, de Gerstheim (Bas-Rhin), fils d'agriculteurs, qui quitte la Dordogne « le cœur gros ». " Je n'avais pas bien envie de rentrer, raconte-t-il, parce que entretemps j'avais connu une fille ${ }^{(12)}$.

Dès le chemin du retour, et jusqu'à leur ville ou village, où l'accueil est chaleureux, les évacués sont l'objet de toutes les attentions de la part de l'occupant. La prévenance des autorités est évidemment calculée, mais elle est appréciable pour des voyageurs inquiets et harassés. D’autant plus que les " rentrants » ne sont pas au bout de leurs surprises. "Nous trouvâmes ce que nous avions redouté de trouver, c'est-à-dire nos maisons pillées, nos meubles disparus ou détruits ", écrit le secrétaire de la mairie de Blodelsheim (Haut-Rhin) ${ }^{(13)}$. L’armée française, armée de la nation, a détruit et pillé, découvre-t-on trop souvent; l'Allemagne, l'ennemie, répare et finance les réparations. La comparaison n'est guère flatteuse pour la France. En même temps circulent des phrases toutes faites auxquelles la matérialité des faits ne peut que donner une certaine consistance: «La France nous a laissé tomber » ${ }^{(14)}$.

Toutefois, même si la République a en effet donné l'impression d'avoir abandonné à leur sort une partie de ses enfants, l'Alsace repeinte en brun n'a rien d'attirant pour la très grande majorité de ceux qui retournent chez eux. En septembre 1940, un lycéen strasbourgeois quitte Périgueux pour revenir dans son foyer avec ses parents. "Sans m'en douter vraiment, je venais, comme bien d'autres, d'entrer dans la nasse, déclaret-il. Piégé et transporté sur une autre planète, c'est du moins l'impression que j’en ai immédiatement eu " ${ }^{(15)}$. Pour les plus anciens le désenchantement est à la mesure de leur naïveté. "Nos parents n’avaient pas de mauvais souvenirs de leur jeunesse allemande, ils étaient nés sous le régime du Kaiser c'est vrai, mais ce n'était pas une dictature. Ils s'imaginaient que ce serait pareil » ${ }^{(16)}$.

10 Jacques Gandebeuf, Claudius Thiriet, La Parole retrouvée: près de 200 Mosellans racontent leur vie entre 1940 et 1945, Metz, 1998, p. 292-293.

11 TO, Jean Amos, Strasbourg, $1^{\text {er }}$ décembre 2001.

12 TO, Alfred Bapst, Gerstheim, 21 avril 2008.

13 Archives départementales du Haut-Rhin (ADHR), $1441 \mathrm{~W}$ 1, Enquête sur l'histoire de l'occupation et de la libération de la France. Commune de Blodelsheim, 20 mars 1951.

14 Id., Enquête... Commune de Feldkirch, s.d. (1950 ou 1951).

15 Jean JARDINÉ,...Alias Gartner. Un itinéraire alsacien, dactylographié, s.d., p. 80.

16 TO, Alfred Bapst, 21 avril 2008. 


\section{Le maillage politique totalitaire ${ }^{(17)}$}

Pour éviter la fâcheuse impression que pourraient ressentir les Alsaciens et Lorrains mosellans à leur retour, celle de se retrouver dans une région conquise par une armée étrangère, et pour aller dans le sens de l'idéologie officielle d'un retour à un état de choses naturel interrompu en 1918 , les nazis utilisent d'abord les autonomistes ${ }^{(18)}$, censés incarner la part la plus saine et la plus fidèle de la population. Ce sont certains de ces autonomistes et des «Alsaciens-Lorrains du Reich » (ceux partis d'eux-mêmes ou expulsés en Allemagne en 1918-1919) qui mettent en place le Secours alsacien (Elsässischer Hilfsdienst ${ }^{(19)}$ - EHD) pour l'accueil des évacués. Son principal créateur, Robert Ernst (1897-1980) ${ }^{(20)}$, pense pendant un court moment pouvoir le transformer en un mouvement régionaliste à l'intérieur du Reich, mais il se heurte rapidement au jacobinisme nazi. Pour le Gauleiter Wagner, en effet, « les Alsaciens sont tout juste bons à être des auxiliaires des Allemands, et c'est dans ce but que le Hilfsdienst est utilisé " (21).

Au lendemain du manifeste des Trois-Épis (Haut-Rhin) du 18 juillet 1940, par lequel, sur l'inspiration d'Ernst, les Nancéens (Nanziger) ${ }^{(22)}$ ont demandé l'annexion de l'Alsace-Moselle au III Reich (sans doute afin de pouvoir continuer à avoir voix au chapitre), l'EHD s'efface parce que les tendances cléricales et particularistes de beaucoup de ses membres sont incompatibles avec l'idéologie nationale-socialiste; il est finalement dissout en avril 1941. L'histoire retient donc surtout ce manifeste comme un acte de trahison envers la France - qui plus est complètement inutile -, et les " patriotes alsaciens » déchantent et fulminent.

Engagé volontaire dans l'armée allemande en 1914, grand blessé de guerre (comme pilote d'avion), Ernst est un Alsacien authentique, fils du pasteur de Hurtigheim (BasRhin). Installé dans la République de Weimar, il a été l'un des fondateurs de l'Union des Alsaciens-Lorrains de souche (Alt-Elsaß-Lothringische Vereinigung) et il est entré au comité directeur de l'Institut scientifique des Alsaciens-Lorrains du Reich (Wissenschaftliches Institut der Elsaß-Lothringer im Reich) à Francfort ${ }^{(23)}$. Otto Meissner, né à Bischwiller (Bas-Rhin), parent par alliance de Kléber; Fritz Bronner ${ }^{(24)}$, né à Riquewihr

17 Nous empruntons cette judicieuse formule à Alphonse Irjud ("Une Résistance éclatée dans un maillage totalitaire ", dans Alfred WAHL, Les Résistances des Alsaciens-Mosellans durant la Seconde Guerre mondiale 1939-1945, Metz, 2006, p. 31-38).

18 Voir Philip Bankwitz, Les Chefs autonomistes alsaciens 1919-1947, Strasbourg, 1980.

19 Voir L. Kettenacker, «La politique de nazification en Alsace » (note 2), p. 104-117.

20 Oberstadtkommisar (maire) de Strasbourg (puis le 14 février 1942 Oberbürgermeister - maire du "grand Strasbourg ", après annexion des communes périphériques) et Generalreferent (rapporteur général) du Gauleiter. Voir L. Kettenacker, «La Politique de nazification en Alsace » (note 2), p. 76-90.

21 Eugène RiEdWEg, 1939-1945. Strasbourg: ville occupée. La vie quotidienne dans la capitale de l'Alsace durant la Seconde Guerre mondiale, Steinbrunn-le-Haut, 1982, p. 85.

22 Frange germanophile des autonomistes emprisonnés en 1939 par les autorités françaises à Nancy. L’un d'eux, Karl (Charles) Roos, a été fusillé pour espionnage à Champigneulles (Meurthe-et-Moselle) le 7 février 1940.

23 Voir Irmgard Grünewald, Die Elsaß-Lothringer im Reich 1918-1933. Ihre Organisationen zwischen Integration und "Kampf und die Seele der Heimat", Francfort/Berne, 1984.

24 Directeur de l'école normale de Colmar pendant l'annexion de fait (Charles Béné, L'Alsace dans les griffes nazies, vol. 6, Raon-l'Étape, 1984, p. 58). 
(Haut-Rhin), et le général Scheüch ${ }^{(25)}$, né à Sélestat (Bas-Rhin), dernier ministre de la Guerre de Guillaume II (et cousin du général français Reibell), comptent parmi les figures les plus marquantes de ce groupe ${ }^{(26)}$. Beaucoup de ceux qui sont encore vivants regagnent le pays natal en 1940, avec des Allemands nés dans le Reichsland. Parmi ces « revenants » ${ }^{(27)}$, Theodor Berkelmann (1894-1943), né au Ban-Saint-Martin, un quartier de Metz, haut dirigeant de la SS, chargé de la politique de colonisation en Lorraine ${ }^{(28)}$.

Après la très brève période de séduction, le rouleau compresseur se met en marche. Sous l'autorité du Gauleiter se trouvent placés les chefs politiques de rangs inférieurs (membres du Korps der politischen Leiter) : Kreisleiter (sorte de sous-préfet politique) ${ }^{(29)}$, Ortsgruppenleiter (chef d'arrondissement de police politique), Zellenleiter (chef de cellule), Blockleiter (chef d'un groupe d'immeubles ou de maisons) et Mitglieder (membre du parti). En beaucoup d'endroits, les opportunistes surgissent, comme François Haegelin, viticulteur à Orschwihr (Haut-Rhin), nommé Oberbauernführer (chef de plusieurs groupements locaux d'agriculteurs), qui croit « le moment venu pour devenir le tyran du village " ${ }^{\left({ }^{30}\right)}$. L'entrée dans le parti est cependant réservée à une "élite » de militants éprouvés, passés par un "sas " appelé Opferring (Cercle du sacrifice) en Alsace, institué le $1^{\text {er }}$ octobre 1940 - et, dans le Gau voisin, Deutsche Volksgemeinschaft Lothringen (Communauté du peuple de souche allemande de Lorraine) ${ }^{(31)}$, créée dès le 20 août - , sorte d'état probatoire ${ }^{(32)}$.

La NSDAP (introduite en Alsace en mars 1941 seulement, et dont les premiers membres alsaciens ne sont reçus que le 25 janvier 1942) quadrille la société, composée dans chaque Gau à la fois de Reichsdeutschen (citoyens du Reich dans ses frontières de 1937, qui jouissent de la plénitude des «droits » et des devoirs, dont le service militaire) et de Volksdeutschen (personnes de «souche germanique », mais qui ne peuvent

25 Voir Jean-François Thull, « Une carrière dans l’armée allemande: Heinrich Scheüch (1864-1946), un général alsacien dans l'armée de Guillaume II », in: Jean-Noël Grandhomme (dir.), L'Alsace et la Grande Guerre, Revue d'Alsace, 139 (2013), p. 147-153.

26 L. Kettenacker, "La politique de nazification en Alsace " (note 2), p. 79.

27 Expression qui désigne les Alsaciens-Lorrains émigrés en France ou leurs descendants rentrés dans leur région d'origine après 1918.

28 Bernard et Gérard Le MAREc, Les Années noires. La Moselle annexée par Hitler, Metz, 1990, p. 145-146.

29 La liste des Kreisleiter d'Alsace est la suivante (nous conservons la forme germanisée des noms, même pour les Alsaciens de souche): Altkirch - Josef Fiterer, Colmar (Kolmar) - Konrad Glas, Erstein Johann Bender, Guebwiller (Gebweiler) - Alexander Krämer, Haguenau (Hagenau) - Renatus Hauss, Molsheim - Edmund Nussbaum, Mulhouse (Mülhausen) - Hans Peter Murer, Ribeauvillé (Rappoltsweiler) - Walter Kirn, Saverne (Zabern) - Rudolf Lang, Sélestat (Schlettstadt) - Heinrich Sauerhöfer, Strasbourg (Straßburg) - Hermann Bickler, Thann - Karl Eschle, Wissembourg (Weißenburg) - Reinhold Lawnick (Archives municipales de Strasbourg [AMS], 207 MW 5, Note du Chef der Ziverwaltung, 10 janvier 1941).

30 ADHR, $1441 \mathrm{~W}$ 6, Commission consultative de sécurité publique de l'arrondissement de Guebwiller, PV de la séance du 29 mars 1945.

31 Eugène Schaeffer, L'Alsace et la Lorraine (1940-1945). Leur occupation en droit et en fait, Paris, 1953, p. 91.

32 Paul Eschвасн, Pleurs et grincements de dent. Un adolescent d'Alsace sous l'Occupation, Mulhouse, 1998, p. 32. 
obtenir la citoyenneté allemande qu'individuellement, pour services rendus), parfois ironiquement surnommés Beutedeutschen (Allemands «par butin ») ${ }^{(33)}$.

Ce statut de Volksdeutsch a également été attribué (ou plutôt imposé) dans les territoires annexés à certains Slovènes, Tchèques et Polonais catalogués «d'ascendance allemande ", aux Luxembourgeois, aux Belges d'Eupen et de Malmédy, aux Sudètes, aux Allemands de Moravie; dans les territoires occupés, satellisés et alliés: aux Allemands du Danemark, des Pays baltes, de la Volga, de Hongrie, de Serbie, de Slovaquie, du Tyrol du Sud (Haut-Adige), de Transylvanie, de Bessarabie; un statut proche existe pour les pays d'émigration (Auslandsdeutschen). Pour les pangermanistes les plus exaltés ce sont d'ailleurs tous les peuples d'origine germanique qui ont vocation à intégrer un jour le Grand Reich. Dans ce cadre, l'Alsace-Moselle se trouve sur le front pionnier de la germanisation ${ }^{(34)}$. Les habitants des régions annexées connaissent toutes les étapes de la mise au pas, et ceux de la Lorraine française, du Nord, de la Champagne, des Ardennes, de la Franche-Comté et de la Bourgogne, elles aussi «vieille terres franques, alémaniques ou burgondes », ne perdent rien pour attendre.

\section{Défrancisation, rage " purificatrice » et germanisation}

Dès la fin de juin 1940 un grand « coup de balai » est donné. Les murs sont couverts de l'affiche: Hinaus mit dem welschen Plunder! (À la porte le foutoir français!) ${ }^{(35)}$. Comme raison de l'incroyable débandade des militaires et des fonctionnaires français - qui a choqué tout particulièrement des populations alsaciennes et lorraines mosellanes fondamentalement légalistes et respectueuses des corps constitués -, les nazis avancent une explication "imparable ", qu'ils fournissent aux Sélestadiens: "Si vous êtes dans de mauvais draps maintenant, c'est la faute de vos Juifs! " ${ }^{(36)}$. Cet antisémitisme forcené heurte en général les populations. «Les lieux de culte israélites sont l'objet d'une haine insensée, s'indigne Robert Bour. La synagogue de Metz est profanée, pillée et sert de débarras. Quant à celle de Sarreguemines, elle est détruite à coups d'explosifs. Le cimetière juif de Thionville est nivelé et transformé en terrain de culture ${ }^{(37)}$.

Vient ensuite le temps des expulsions, massives en Lorraine, plus « ciblées » en Alsace (militants des partis nationalistes, membres du Souvenir français, engagés volontaires dans l'armée française en 1914-1918 ${ }^{(38)}$, Français de l'Intérieur, Juifs, romanichels, " asociaux ", étrangers, avec toujours des exceptions). En tout, ce sont 270000 personnes qui sont concernées entre août 1940 et le début de 1942, auxquelles il faut ajouter 20000

33 Joseph Freymann, Mon vécu de guerre, 1940-1945, Illkirch-Graffenstaden, s.d., p. 10.

34 Voir Jean-Marc Dreyfus, " Germanisierungspolitik im Elsass 1940-1945 », in: Jerzy KochAnOwsKi, Maike SACH (dir.), Die "Volksdeutschen" in Polen, Frankreich, Ungarn und der Tschechoslowakei. Mythos und Realität, Osnabrück, 2006, p. 205-223.

35 ADHR, 1441 W 3, Enquête... Commune d'Oberentzen, 23 août 1950. - TO, Alphonse Bronner, Reichstett, 5 août 2002 .

36 Marie-Joseph Bopp, Ma ville à l'heure nazie. Colmar 1940-1945, Strasbourg, 2004, p. 27.

37 Robert Bour, Un Lorrain dans la Kriegsmarine, Paris, 1977, p. 42.

38 Comme l'ouvrier Auguste Muller, né en 1889, "porté par les Allemands sur la liste des expulsés dès le commencement de l'occupation " (Archives départementales du Bas-Rhin [ADBR], 297 D 15, Comités d'épuration, liste de victimes du nazisme, s.d.). 
départs volontaires clandestins ${ }^{(39)}$. Le moment des expulsions « favorise la confusion et permet l'assouvissement des rancunes personnelles, des haines, des vengeances ${ }^{(40)}$.

Les vides sont en partie comblés par l'installation de colons (Siedler ${ }^{(41)}$ ), parfois venus de l'autre extrémité de l'Europe (comme à Hesse (Moselle), ces Allemands de Bessarabie déplacés en 1940 en vertu des clauses secrètes du pacte germano-soviétique qui ont attribué cette région à l'URSS) ou parfois de beaucoup plus près : les habitants de dix-huit villages de la région dialectophone de Bitche (Bitcherland), où les Allemands ont décidé une grande extension d'un camp militaire, réimplantés en Moselle francophone.

Si le "Nancéen » revient dans sa région en héros (éphémère) de l'ordre nouveau, l'Alsacien ou le Lorrain mosellan rétifs à la germanisation (Reichsfeindliche, ennemi du Reich, ou Politischunzuverlässige, politiquement suspect) - et même à l'occasion l'opposant allemand ${ }^{(42)}$ - sont quant à eux envoyés pour y être " convertis » ou " rééduqués » (umgeschult) dans les camps de Schirmeck-La Broque (Bas-Rhin) ${ }^{(43)}$, Gaggenau (Bade), Schelklingen (Wurtemberg) ou encore à la prison d'Ensisheim (Haut-Rhin), au fort de Queuleu ${ }^{(44)}$, au grand séminaire réquisitionné de Metz, ainsi que dans les prisons de Sarreguemines et de Sarrebourg et au camp de Woippy (Moselle) ${ }^{(45)}$. Les motifs les plus divers peuvent conduire à ces internements et, selon le lieu, le moment ou la personnalité des responsables locaux du parti, une même infraction peut avoir des conséquences anodines ou dramatiques.

Paul Betsch, qui habite La Broque, voit parfois des prisonniers venir à l'atelier de son père pour y chercher de la peinture. «Ceux qui avaient commis les délits les plus graves allaient au Struthof. Nous avions entendu parler de ce camp déjà pendant la guerre, mais on ne s'en approchait pas " ${ }^{(46)}$. Des femmes aussi sont détenues à Schirmeck, comme une voisine de celui qui deviendra le notaire Camille Bilger, veuve d'un capitaine de l'armée française tué en 1940, et mère de trois enfants, qui y passe trois mois, dénoncée par « une des filles d'une famille germanophile » pour avoir écouté la radio suisse ${ }^{(47)}$. L'un des internés, Gustave Bato, a été arrêté le 15 novembre 1941 pour avoir chanté Les Gars de la marine dans un café de Soultz (Haut-Rhin) ${ }^{(48)}$. D’une manière générale, c'est toute trace de culture française et démocratique qui doit disparaître du territoire

39 La Voix des groupements GERAL-PRAF, juillet 1997, $\mathrm{n}^{\circ} 319$.

40 R. Bour, Un Lorrain dans la Kriegsmarine (note 37), p. 29.

41 Voir B. et G. Le Marec, Les Années noires (note 28), p. 132-135.

42 Jean-Paul Lingelser, "Lipsheim, Fegersheim et Ohnheim sous la domination allemande, 19391945 », Annuaire de la Société d'histoire des Quatre cantons, n²2, 2004, p. 57.

43 Voir «Il y a cinquante ans: la libération du camp. Le camp d'internement de Schirmeck, Sicherungslager Vorbruck ", numéro thématique de la revue L'Essor, n 65, décembre 1994. - Jacques GRANIER, Schirmeck. Histoire d'un camp de concentration, Strasbourg, s.d. et Ch. BÉné, L’Alsace dans les griffes nazies (note 24), vol. 5, 1980, p. 45-82.

44 Voir Léon Burger, Tragédies mosellanes. Le fort de Queuleu à Metz, Metz, 1973.

45 Voir Marcel Neigert, « La Répression allemande en Moselle (1940-1945) ", Revue d'histoire de la Seconde Guerre mondiale, $\mathrm{n}^{\circ}$ 105, janvier 1977, p. 79-100. - Id., Internements et déportation en Moselle 1940-1945, Metz, 1978. TO, Paul Betsch, La Broque, 18 décembre 2008.

ADHR, 1441 W 4, Plainte adressée au président des FFI de la région de Soultz, s.d. 
« recouvré » par l'Allemagne. Dans de nombreux endroits les habitants sont donc invités à déposer entre les mains des autorités leurs livres, insignes, décorations, uniformes, livrets militaires français ${ }^{(49)}$. Dans bien des cas pourtant, comme à Pulversheim (Haut-Rhin), le corps enseignant, le personnel communal ou même des particuliers réussissent à mettre "en lieux sûrs un nombre appréciables d'ouvrages, ainsi que les cartes géographiques, tous les drapeaux tricolores et le buste de La République » ${ }^{(50)}$.

Sont ensuite organisés des bûchers de livres (Bücherverbrennung) ${ }^{(51)}$. Ces autodafés ont généralement lieu à l'occasion de la fête du solstice d'hiver (Sonnenwendfeier) ${ }^{(52)}$, fête païenne qui doit concurrencer Noël. Sont simultanément implantées un peu partout des bibliothèques communales (Volksbücherei) ${ }^{(53)}$ pour diffuser la vulgate nazie ${ }^{(54)}$. En général, elles se composent essentiellement de «volumes de propagande, destinés à la jeunesse, où on loue l'héroïsme des combattants allemands " ${ }^{(55)}$. Des séances de cinéma avec surtout les actualités (Wochenschau) «où l'on montre les victoires allemandes » ou encore le classique de l'antisémitisme Jud Süss (Le Juif Süss) ${ }^{(56)}$ et des représentations théâtrales ${ }^{(57)}$ sont également organisées. La NSDAP propose encore des Kulturabende (soirées culturelles) avec concerts et conférences (sur les thèmes: Die grosse Wende - Le Grand tournant ${ }^{(58)}$ et Adolf Hitler rettet Europa - Adolf Hitler sauve l'Europe $\left.{ }^{(59)}\right)$. La propagande allemande se substitue donc à l'éducation française.

En dehors des personnes ayant un poste de confiance ou de direction dans les diverses organisations, et d'un certain nombre de ralliés ou d'opportunistes, la population rechigne à participer aux démonstrations, comme le Kreistag (Jour de l'arrondissement) ou le Großkundgebung (Grande manifestation) ${ }^{(60)}$. Alors, à Ribeauvillé, pour le troisième Kreistag, les 25 et 26 septembre 1943, tous les membres des organisations des environs sont convoqués en uniforme. Les Blockleiter font commerce de drapeaux à croix gammée avec ce slogan: "Aucune maison de notre ville sans un drapeau de la Grande Allemagne! » ${ }^{(61)}$. En Moselle les Allemands organisent des

49 Id., 1441 W 3, Enquête... Commune de Jungholtz, s.d. (1950 ou 1951).

50 Id., Enquête... Commune de Pulversheim, s.d. (1950 ou 1951).

51 Comme celle organisée sur la Karl-Roos-Platz (place Kléber) à Strasbourg pour la Noël de 1940 (SNN, 27 décembre 1940).

52 Voir GenevièveHerberich-MArx, FreddyRAPHAËL, «Unecroisadedepurification culturelle. Les autodafés du solstice d'hiver dans l'Alsace annexée (1940) », Revue des sciences sociales de la France de l'Est, 21 (1994), p. 61-70.

53 Marc Schweyer, "Aspects dela politique nationale-socialiste en Alsace. La Bibliothèque municipale de Strasbourg sous l'Occupation (1940-1944) », Saisons d'Alsace, été-automne 1971, p. 489-490.

54 ADHR, 1441 W 3, Enquête... Commune d'Oberentzen, 23 août 1950.

55 Id., 1441 W 2, Enquête... Commune de Hattstatt, 18 décembre 1950.

56 Id., 1441 W 1, Enquête... Commune de Feldkirch, s.d. (1950 ou 1951).

57 Id., 1441 W 2, Enquête... Commune d'Issenheim, s.d. (1950 ou 1951).

58 Prononcée par exemple par les autonomistes Schall, Hauss et Keppi à Haguenau, le 28 juillet 1940 (André WAGNER, «Chronique des années 1938-1946 », Études haguenoviennes, 1994, t. XX, p. 31).

59 ADHR, 1441 W 1, Enquête... Commune de Bollwiller, s.d. (1950 ou 1951).

60 Id., 1441 W 3, Enquête... Commune de Pulversheim, s.d. (1950 ou 1951).

61 «Kein Haus unserer Stadt ohne eine Fahne Groß-Deutschlands! ( «Des Années noires à la Libération, 1939-1945 ", Bulletin du Cercle de recherche historique de Ribeauvillé et environs, $\mathrm{n}^{\circ}$ 8, décembre 1994, p. 23). 
commémorations ostentatoires chaque année pour célébrer les victoires allemandes de 1870 autour de Metz ${ }^{(62)}$.

\section{Le vandalisme et les persécutions linguistiques}

Les attaques contre la culture locale et nationale sont frontales. Les Allemands entreprennent la défrancisation de l'Alsace-Lorraine et de ses habitants (Entwelschung von Land und Leute) selon la doctrine de la Rückdeutschung (re-germanisation), l'Alsace et la Lorraine mosellane ayant été, selon eux, arrachées à la sphère politique germanique par les " rapts " successifs des rois de France, surtout de Louis XIV. Dans ce cadre, tout ce qui rappelle l'appartenance du pays à la culture française doit disparaître. Les monuments aux morts, qui sont souvent détruits ou germanisés, constituent bizarrement l'une des cibles principales des nazis, alors qu'ils ont été élevés en mémoire de soldats très majoritairement morts sous l'uniforme de l'armée allemande ${ }^{(63)}$. À Metz, " amputé de ses bas-reliefs, [il] est accommodé à la sauce germanique, il porte en gothique: "Sie starben für das Reich" [Ils sont morts pour le Reich] » ${ }^{(64)}$. Dans bien des cas donc le monument n'est que partiellement détruit.

Les statues et monuments "français » (comme ceux de Kléber à Strasbourg, de Lafayette à Metz ou de Rapp à Colmar) sont bien évidemment eux aussi démontés ou détruits. Les noms des communes sont modifiés (Thionville redevient Diedenhoffen, Ribeauvillé Rappoltsweiler, Sélestat Schledstadt) et également ceux des rues ${ }^{(65)}$ : à Sarrebourg la rue Jeanne d'Arc se mue en Richard-Wagnerstraße. Les nouveaux maîtres importent donc leurs références culturelles ${ }^{(66)}$. En dehors de zones francophones d'Alsace et de Moselle, et des grandes villes, les Allemands n'éprouvent guère de difficulté à faire disparaître le français, peu parlé dans la vie courante. La langue prohibée est remplacée par le haut allemand car le jacobinisme nazi s'accommode mal du dialecte, d'autant plus qu'il a assimilé un certain nombre d'expressions françaises (bouchour, "bonjour »; ou merci) ${ }^{(67)}$. De nombreuses anecdotes montrent la résistance passive d'une grande partie de la population à ces mesures.

Les Allemands vont bien au-delà de ce qu'avait exigé la dictature militaire en 1914. Ils imposent même des changements de prénoms et de noms de famille ${ }^{(68)}$, avec toutefois

62 Monique SARY, «La Vie à Metz sous l'occupation nazie (1940-1944) », in: François-Yves Le MoIGNE, Moselle et Mosellans dans la Seconde Guerre mondiale, Metz, 1983, p. 162.

63 Jean-Noël Grandhomme, "Un Aspect de la "mise au pas” de l'Alsace-Moselle annexée de fait: la destruction des monuments aux morts de 1914-1918 par les nazis pendant la Seconde Guerre mondiale », in: Stéphane Benoist, Anne Daguet-Gagey, Christine Hoët-Van Cauwenberghe et Sabine Lefebvre, Mémoires partagées, mémoires disputées: écriture et réécriture de l’histoire, Metz, 2010, p. 253-272.

64 R. Bour, Un Lorrain dans la Kriegsmarine (note 37), p. 32.

65 Gérard Michaux, "Sarrebourg à “l’heure allemande” », in: F.-Y. LE Moigne, Moselle et Mosellans (note 62), p. 199.

66 Une des transformations les plus ridicules, qui fait bien rire sous le manteau, est celle de la principale rue commerçante de Mulhouse, la rue du Sauvage, en Adolf-Hitler-Straße (E. RiedwEg, 1939-1945: Mulhouse ville occupée, Steinbrunn-le-haut, 1981, p. 48).

67 ADHR, 1441 W 1, Enquête... Commune de Feldkirch, s.d. (1950 ou 1951).

68 Bourgeois se mue en Burg ou encore Petit en Klein (ADHR, 1441 W 3, Enquête... Commune de Pulversheim, s.d. [1950 ou 1951]). - Voir aussi AMS, 207 MW 13. 
de nombreuses exceptions, comme cette famille au patronyme typiquement breton: Gargoët ${ }^{(69)}$, ou encore ces Mosellans nommés Blondlot, Chevrier, Grandhomme, Meunier, qui conservent leur patronyme tout au long de la guerre. Parfois les modifications touchent à l'absurde. Le port du béret (Franzosenmütze ou Gehirnverdunkelungkappe) est interdit car il « obscurcit le cerveau " selon le Kreisleiter de Colmar ${ }^{(70)}$; en d'autres endroits il est dénoncé comme " un couvre-chef juif » ${ }^{(71)}$. Les coupes de cheveux sont elles aussi surveillées. «Pour moi, je garde le cheveu long et, comble d'indiscipline, les pattes sur les joues », raconte Robert Bour ${ }^{(72)}$.

Les journaux français disparaissent, remplacés en Alsace par les Straßburger Neueste Nachrichten ${ }^{(73)}$, le Kolmarer Kurier, le Mülhauser Tagblatt; et en Moselle par la National Sozialistiche Zeitung Westmark ou la Metzer Zeitung (puis Metzer Zeitung am Abend), simples courroies de transmission des services de propagande du nouveau régime. Les fonctionnaires alsaciens sont envoyés outre-Rhin pour des stages de " recyclage » ou de "mise aux normes" (Umschulung). Sont particulièrement touchés les cadres de la SNCF ${ }^{(74)}$, devenue Reichsbahn; des PTT, devenues Reichspost; ou les instituteurs et institutrices. La vie économique est elle aussi prise en main par l'État. Enfin, les Allemands procèdent à des simplifications administratives en créant de grandes métropoles à Mulhouse, Metz, Strasbourg; et en regroupant plusieurs villages ${ }^{(75)}$.

\section{La pression politique}

Tous les arguments sont bons pour attirer des autochtones jugés intéressants vers la NSDAP. Convoqué chez son Blockleiter, Robert Bour, homme jeune et dynamique, entend parler de son passé de marin français. "En août 1940, j’ai drôlement pensé à toi. Mers el-Kébir, Dakar, Alexandrie, tu y étais, non? [...] Quelle honte! Les Anglais, quels salauds! Et dire qu'il y a des Français qui marchent avec eux. Mais toi, t'es un type droit! T’as compris, non? » Devant le silence de son interlocuteur, le responsable nazi alterne promesses et menaces, mais n'obtient aucun résultat. En conséquence Robert Bour est renvoyé dès le lendemain de son emploi. Conscient d'avoir été imprudent, il décide de déménager. «De l'autre côté de la rue, c'est un autre bloc. Et le Blockleiter y est moins agressif » ${ }^{(76)}$.

Comme dans les autres territoires annexés en Europe, les organisations nazies sont importées en Alsace-Moselle, dès le 15 octobre $1940^{(77)}$. Elles s'implantent rapidement

69 TO, Simone Gargoët-Nevels, 25 novembre 2002 (entretien réalisé par Laure Balzano-Dupuich). Cette famille était fixée depuis longtemps à Sainte-Marie-aux-Mines (Haut-Rhin), le père avait fait la Grande Guerre dans l'armée allemande.

70 Pierre Rigoulot, L’Alsace-Lorraine pendant la guerre 1939-1945 (1997), Paris, 1998, p. 37.

71 ADHR, 1441 W 1, Enquête... Commune de Biltzheim, 9 juillet 1950.

72 R. Bour, Un Lorrain dans la Kriegsmarine (note 37), p. 34.

73 Voir Isabelle Bogen, «Les SNN, sept colonnes de propagande à la “une” ", Saisons d'Alsace, n 114: 1941, la Mise au pas, 1991/1992, p. 51-62.

TO, André Claus, Lingolsheim, 9 septembre 2002.

TO, Paul Betsch, 18 décembre 2008.

R. Bour, Un Lorrain dans la Kriegsmarine (note 37), p. 83-85.

SNN, 17 octobre 1940, citées par Mathieu DANNER, Scherwiller et ses habitants, 1939-1945. L'histoire d'un village alsacien pendant la Seconde Guerre mondiale, Maîtrise, Strasbourg II, 2003, p. 57. 
jusqu'au fond des campagnes, rencontrant parfois de farouches résistances, comme à Réguisheim (Haut-Rhin), où aucune n'existe autrement que sur le papier, ce qui entraîne une lourde répression ${ }^{(78)}$, ou encore à Biltzheim (Haut-Rhin), village d'agriculteurs et de mineurs de potasse, où « la population a observé une attitude irréprochable, de sorte qu'aucun habitant n'a été poursuivi pour faits de collaboration à la Libération ${ }^{(79)}$. Plusieurs formations paramilitaires dépendent directement du parti (Gliederungen der Partei): SA (Sturmabteilung), SS (Schutzstaffel), NSKK (Nationalsozialistisches Kraftfahrkorps - corps motorisé national-socialiste), HJ (Hitlerjugend), NSFK (Nationalsozialistisches Fliegerkorps - corps national-socialiste d'aviation ${ }^{(80)}$, qui attire, à l'image des autres organisations, son lot d'Alsaciens ${ }^{(81)}$ et de Lorrains ${ }^{(82)}$, avant tout passionnés d'aviation ou de modélisme $\left.{ }^{(83)}\right)$.

Le quadrillage de l'Alsace et de la Moselle par la NSDAP et ses organisations est bien supérieur à celui des autres régions (un Block pour 99 habitants en Alsace contre un pour 162 au Pays de Bade $\left.{ }^{(84)}\right)$. Pour pouvoir continuer à exercer dans l'administration les fonctionnaires doivent produire un certificat d'hérédité (Abstammungsnachweis) ou d'aryanité (Ahnenpass ${ }^{(85)}$ ou Ariernachweis) ${ }^{(86)}$. Les employés de l'État et assimilés sont poussés à entrer dans la NSDAP et à y accepter des responsabilités. Les femmes sont sollicitées par le Secours d'hiver (Winterhilfsdienst), division de l'Agence des femmes allemandes (Frauenwerk), la NSF (Nationalsozialistische Frauenschaft - Union nationale-socialiste des femmes $\left.{ }^{(87)}\right)$ ou encore par l'Agence de secours "Mère et Enfant » (Hilfswerk Mutter und Kind), qui dépend de la NSV (Nationalsozialistische Volkswohlfahrt - Ligue nationale-socialiste pour le bien-être du peuple $\left.{ }^{(88)}\right)$. Les nazis ont le champ entièrement libre puisque le 22 mars 1941 toutes les associations caritatives confessionnelles ou autres ont été interdites ${ }^{(89)}$. Si la grande majorité des Alsaciennes

78 ADHR, 1441 W 3, Enquête... Commune de Réguisheim, 17 février 1951.

79 Id., $1441 \mathrm{~W}$ 1, Enquête... Commune de Biltzheim, 9 juillet 1950.

80 Voir "National Socialist Flyers' Corps », in: Friedemann Bedürftig, Christian Zentner (dir.), The Encyclopedia of the Third Reich, New-York, 1997, p. 627-628.

81 ADBR, 544 D 238, Comité départemental d'épuration, fiche d'un instituteur (non sanctionné), 27 mars 1946.

82 ADM, 1486 W 701, Dossier d’A. T. (né à Felsberg (Sarre) en 1905, marié à une Lorraine, naturalisé français). Notice de renseignements concernant un individu jugé dangereux pour la défense nationale ou la sécurité publique, 30 mai 1945.

83 Émile Hettler, Jean-Paul Grasser, Études haguenoviennes, t. XIII: Haguenau dans la tourmente de 1939-1945, vol. 1, 1987, p. 168.

84 Eugène Riedweg, Les Malgré-nous, Mulhouse, 1995, p. 28.

85 Voir Christian Wolf, «Le Destin curieux de l'Ahnenpass. Le certificat d'aryanité est devenu un outil généalogique ", Saisons d'Alsace (note 73), p. 125-130.

86 Certificat sous forme d'arbre généalogique demandé à un postulant à la SA, in: Michèle HerzBERG, Francis Lichtlé, Roland Oberlé, Batailles d'Alsace 1939-1945, Strasbourg, 1988, p. 142-143.

87 Voir Jill Stephenson, The Nazi Organization of Women, New-York, 1981 et Women in Nazi Society, New-York, 1985.

88 Voir Sophie Friederich, La Nationalsozialistische Volkswohlfahrt à Strasbourg de 1940 à 1944, Maîtrise, Strasbourg II, 1999.

89 L. Kettenacker, "La politique de nazification en Alsace. Deuxième partie ", Saisons d'Alsace, nº 68 (1979), p. 20. 
et des Lorraines mosellanes n'adhère à aucune des filiales du parti, certaines trouvent une échappatoire en entrant dans la Croix-Rouge (Deutsches Rotes Kreuz - DRK).

Il existe aussi de nombreuses organisations professionnelles comme le Front allemand du Travail (Deutsche Arbeitsfront - DAF), créé en 1933 après la dissolution des syndicats, la Ligue nationale-socialiste des enseignants et des fonctionnaires (Nationalsozialistischer Lehrer- und Beamtenbund - NSLB), celle des étudiants (Nationalsozialistische deutscher Studentenbund - NSDS), etc. "Pour ne pas être obligé à une activité politique quelconque, détestée de presque tous, écrit le sénateur Médard Brogly, beaucoup d'Alsaciens se faisaient enrôler dans ces organisations professionnelles ${ }^{(90)}$. Sur un ou deux points précis, le régime allemand - comme d'ailleurs celui de la période de la première Annexion - comporte tout de même de bons côtés, admet Robert Bour (que l'on ne peut soupçonner de complaisance pour le nazisme): "L'assurance Accidents Maladie est l'un des systèmes qui, à mon sens, présente quelque avantage sur la législation française de l'époque. [...] De plus, une assurance vieillesse est prévue lors de la retraite à soixante-cinq ans " ${ }^{(91)}$.

La majorité de la population, cependant, ne rejoint aucune organisation, les ralliés n'auraient représenté qu'environ $2 \%$ des habitants ${ }^{(92)}$. Le chiffre est sans doute plus élevé, mais somme toute assez comparable à celui des collaborateurs dans les autres régions françaises. Dans bien des cas l'administration locale est maintenue, le maire d'avant l'annexion de fait acceptant de rester en place comme un " moindre mal " ou un citoyen se dévouant pour remplir une des fonctions liées au parti, à l'exemple de Joseph Ginter à Soultz, "Ortsgruppenleiter ayant fait tout son possible pendant l'occupation allemande afin de soulager la population opprimée en prenant beaucoup de risques à son compte ${ }^{(93)}$.

\section{L'embrigadement de la jeunesse: le cadre scolaire}

Le régime contraint les adolescents, et même les enfants, à entrer dans différentes organisations. La propagande dispensée par le maître d'école (Schulmeister), plus ou moins nazi (tous les établissements confessionnels sont supprimés le 24 mars 1941), se double ainsi d'un endoctrinement spécifique. L'entrée dans les formations paramilitaires nazies apparaît comme le prolongement logique du système d'éducation du III ${ }^{\mathrm{e}}$ Reich.

Dès l'école primaire, en effet, les enfants sont soumis à une propagande militaire. La lecture de certains petits textes leur est imposée afin de les sensibiliser aux différents types de " héros » allemands: Aus dem Tagebuch eines Jagdfliegers (Extrait du journal d'un pilote de chasse) par Goering lui-même, ou Scapa Flow par Günther Prien, le plus grand as de l'arme sous-marine (surnommé le Taureau de Scapa Flow après avoir

90 Médard Brogly, La Grande épreuve. L’Alsace sous l'occupation allemande, 1940-1944, Paris, 1945, p. 69.

91 R. Bour, Un Lorrain dans la Kriegsmarine (note 37), p. 38.

92 Gisèle Rapp-Meichler, Alsace, les années fantômes. Chronique des années de guerre, 1939-1945, Mulhouse, 1993, p. 123.

93 ADHR, 1441 W 6, Commission consultative de sécurité publique de l'arrondissement de Guebwiller, s.d. 
réussi à pénétrer en octobre 1939 dans la base britannique pour y couler le cuirassé Royal Oak) ${ }^{(94)}$.

Dans les établissements scolaires les élèves sont maintenant entourés de camarades allemands, dans des proportions variables, plus nombreux dans les villes. « Les parents étaient fonctionnaires ou militaires, se souvient Benoît Daul. Il y avait aussi des réfugiés, venus pour fuir les villes allemandes bombardées, afin d'être tranquilles. Nous n'avions pas beaucoup de relations avec eux. [...] On ne cherchait pas d'ennuis. Parmi ces Allemands, c'était comme partout, il y en avait des bons et des moins bons. On ne faisait pas de politique ${ }^{(95)}$.

Le corps professoral, lui aussi, a beaucoup changé. Le collège épiscopal de Zillisheim ayant été fermé par les Allemands, Camille Bilger s'est inscrit au lycée d'Altkirch. "Comme on était sous le régime allemand on avait classe jusqu'à 13 heures. Je trouve ce système meilleur que le nôtre! Et l'après-midi on rentrait et on faisait du sport. » La plupart des professeurs sont des Allemands âgés ou exemptés de service pour raison de santé, et quelques professeurs alsaciens «qui ont réussi à se maintenir» ${ }^{(96)}$.

Par la force des choses, les annexés de fait plus âgés sont contraints de fréquenter les universités d'outre-Rhin avant la réouverture de celle de Strasbourg. Jean Amos se dirige vers Heidelberg. "C'était une ville très sympathique et très ouverte aux étudiants. Clermont, où nous étions un peu entassés, ne pouvait souffrir la comparaison. Heidelberg était une ville universitaire depuis plus de quatre cents ans, et la mentalité des gens était tout autre; nous avons été très bien reçus, on ne peut pas dire le contraire, même si nous avions faim, comme tout le monde, et si nous entendions les avions qui passaient ${ }^{(97)}$.

\section{L'introduction de la Hitlerjugend et du Bund Deutscher Mädel (BDM)}

À l'âge de six ans les enfants entrent chez les Pimpfe (gamins). De dix à quatorze ans les garçons font partie du Deutsche Jungvolk et les filles du Deutsches Jungmädelbund, puis de quinze à dix-huit ans de la HJ ou du BDM (Ligue des Jeunes filles allemandes $)^{(98)}$. Le passage du Jungvolk à la HJ est marqué par une grande cérémonie, dite Jugendverpflichtung (serment prêté par la jeunesse), destinée à remplacer la communion solennelle des catholiques ou la confirmation des protestants ${ }^{(99)}$. Les Allemands, même nazis, demeurent attachés aux formes juridiques (ou de simple apparence juridique, en tout cas morales) d'engagement, auxquelles ils donnent un aspect contraignant. En vertu du Führerprinzip, HJ et BDM sont très hiérarchisés ${ }^{(100)}$.

\footnotetext{
94 Deutsches Lesbuch für Volksschulen, vol. 3, Berlin, 1943 cité par Michel WAGNER, « L'Enseignement à Lautenbach sous l'Occupation ", S'Lindeblätt, les cahiers du patrimoine du Haut-Florival, nº 17 (1998), p. 35 .
}

95 TO, Benoît Daul, Strasbourg, 18 janvier 2003.

96 TO, Camille Bilger, 11 juillet 2008.

97 TO, Jean Amos, $1^{\text {er }}$ décembre 2001.

98 Harald Scholtz, «Hitler Youth», in: Bedürftig/Zentner, The Encyclopedia of the Third Reich (note 80), p. 431-435.

99 Marie-Joseph Bopp, L'Alsace sous l'occupation allemande, 1940-1945, Le Puy, 1945.

100 Hans-Christian Brandenburg, Die Geschichte der HJ: Wege und Irrwege einer Generation, Cologne, 1968, p. 168 et 182. - Voir aussi Geneviève Humbert, « Les grandes lignes de la politique allemande de 
École d'endoctrinement, machine de guerre destinée à éradiquer en Alsace-Moselle l'éducation française et à arracher les jeunes âmes malléables aux très influentes Églises, la $\mathrm{HJ}$ et le BDM ont une prégnance variable selon les lieux, la personnalité de leurs dirigeants locaux, les milieux concernés. Leur capacité de persuasion ou de coercition dans un but d'endoctrinement ne sont toutefois pas négligeables. Leur audience est réelle et il est certain que le temps l'aurait élargie. Fils de vaincus, jeté hors du «bocal » traditionnellement très structurant des associations religieuses, sportives, musicales, du scoutisme ${ }^{(101)}$, éloigné de sa famille, le jeune Alsacien ou Mosellan, mélangé à des Allemands, est parfois une proie facile, surtout en ville. La séduction est d'autant plus forte que les activités ouvertes à la jeunesse sont réduites à leur plus simple expression.

Quelques mois à peine après l'annexion de fait, un haut fonctionnaire français replié perçoit le danger et en avertit $M^{\mathrm{gr}}$ Ruch, l'évêque de Strasbourg (réfugié à Périgueux), redoutant " toute l'attirance qu'un uniforme, qu'une espèce de sabre, des jeux guerriers et la possibilité de commander comportent pour un adolescent ${ }^{(102)}$. Les réunions de la $\mathrm{HJ}$ et du BDM, fait remarquer un ex-policier français, sont placées le dimanche matin pour empêcher les adolescents de se rendre aux offices. Beaucoup s'inquiètent en constatant que dans le Vieux-Reich « seule la jeune génération allemande, devenue sourde aux disciplines familiales et religieuses, à force de sport et de matérialisme, accepte joyeusement de sortir de l'enfance pour sauter dans la guerre " ${ }^{(103)}$. À partir du 2 janvier 1942, l'adhésion à la HJ est obligatoire aussi en Alsace ${ }^{(104)}$, et à partir du 4 août en Moselle (mais certains arrivent à y couper ${ }^{(105)}$ ). L'absence aux réunions peut conduire à une suppression de l'allocation familiale ${ }^{(106)}$.

"Si tu veux continuer tes études, il va falloir que tu te comportes différemment», dit son directeur d'école à Camille Bilger qui n'a pas caché son profond ennui lorsqu'on l'a obligé, le 9 novembre 1940, à écouter à la radio le discours prononcé par Hitler à l'occasion de l'anniversaire du putsch de la Brasserie, date importante du calendrier national-socialiste ${ }^{(107)}$. Le régime sanctionne ceux qui refusent de se couler dans le moule en hypothéquant leur avenir. "Quand j’ai voulu m’inscrire à la Karl-RoosSchule (ex-Lycée Kléber), confirme un lycéen strasbourgeois, la première question du directeur a été: “Est-ce que tu es inscrit à la HJ?” Lorsque je lui ai dit que non, il m’a

la jeunesse en Alsace occupée, 1940-1944», Revue d'Alsace, nº 110 (1984), p. 193 et « Capter la jeunesse. Les filets de la Hitlerjugend», Saisons d'Alsace (note 73), p. 185-198.

101 Qui ne peuvent survivre que clandestinement après leur suppression par ordonnances des 16 août, 2 et 3 septembre 1940 (Julien Fuchs, Toujours prêt! Scoutisme et mouvements de jeunesse en Alsace, 1918-1970, Strasbourg, 2007, p. 179).

102 Rapport du Directeur général des services d'Alsace et de Lorraine à Périgueux à M ${ }^{g r}$ Ruch, décembre 1940-janvier 1941, in: René EpP, La Terreur nazie en Alsace (1940-1945), Strasbourg, 2002, p. 90.

103 G.-E. Clément, Avec l'Alsace en guerre (1940-1944), Strasbourg, s.d., p. 59. Ancien directeur de la Banque de France à Strasbourg, G.-E. Clément est le seul haut fonctionnaire français resté en Alsace (jusqu'à son arrestation le 15 septembre 1943 et son emprisonnement à Kehl, au Pays de Bade).

104 SNN, 2 janvier 1942 cité par M. DANNER, Scherwiller et ses habitants (note 77), p. 59.

105 TO, Paul Betsch, 18 décembre 2008.

106 TO, Jeanne Kempf-Burgard, Soultzeren, 29 août 2002.

107 TO, Camille Bilger, 11 juillet 2008. 
répondu: "Tu reviendras quand tu y seras" " ${ }^{(108)}$. Le même traitement est réservé aux adolescentes. "Par l'école, j'ai dû rentrer dans le BDM, raconte Simone Gargoët. Les élèves étaient obligées d'y aller. Ils faisaient du chantage à ce sujet, nous disant que nous n'aurions pas notre Abitur si nous n'allions pas dans leur organisation nazie. [...] Comme j'adorais le sport, j'allais à leur séance de sport de 8 à 10 le soir une fois par semaine. Nous avions aussi des heures de piscine ${ }^{(109)}$.

L'éducation nazie met en effet l'accent sur les " exercices physiques » ou " activités corporelles »(Leibesübungen), selon la terminologie en vigueur, les marches, les camps ${ }^{(110)}$, exercices qui tous mènent plus ou moins directement à la formation militaire nazie. Le but est de créer une communauté de sentiments (Gesinnungsgemeinschaft) par absorption de toutes les activités destinées à la jeunesse. Selon une ancienne tradition commune à de nombreux pays européens (y compris la France de Jules Ferry avec ses bataillons scolaires), le régime voit dans tous les sports, ou presque, un moyen de préparer le service militaire (Wehrertüchtigung ${ }^{(111)}$ ), tout particulièrement, bien sûr, par les exercices de tir ${ }^{(112)}$ et d'ordre serré (défilé, garde à vous). Les cours sont aussi très orientés: «On nous expliquait un peu ce qu'étaient l'infanterie, l'aviation, la Kriegsmarine, tout ça... ", déclare André Claus ${ }^{(113)}$.

L'une des missions de la $\mathrm{HJ}$ est d'organiser des quêtes pour améliorer les conditions d'existence des soldats pendant la mauvaise saison. Autour des troncs « une bande papier précise la destination de l'obole "volontaire"! Winterhilf ${ }^{(114)}$, Deutsches Rotes Kreuz, Soldatenheim [Foyer du Soldat], etc. ». Une autre mission réservée aux HJ est la chasse aux doryphores ${ }^{(115)}$. Comme toutes les activités concernant la jeunesse sont passées sous le contrôle du NSDAP, la HJ comprend, entre autres, des sections spécialisées dans la conduite des motos et des automobiles $(\text { Motor-HJ })^{(116)}$, l'aviation par le vol à voile $(\text { Flieger-HJ })^{(117)}$, la marine $\left(\right.$ Marine-HJ) ${ }^{(118)}$, introduites en Alsace à la fin de $1941^{(119)}$.

Les jeunes filles du BDM sont aussi astreintes à une participation à l'effort de guerre: dans le val de Villé (Bas-Rhin), récolte de digitales pour fabriquer des médicaments pour les soldats ${ }^{(120)}$; à la Erwin-von-Steinbach-Oberschule (ex-Lycée Fustel de Coulanges) à

108 TO, André Claus, 9 septembre 2002.

109 TO, Simone Gargoët-Nevels, 25 novembre 2002.

110 TO, André Claus, 9 septembre 2002.

111 Voir Gilbert Krebs, «Une Révolution de la jeunesse? », in: Françoise Knopper, Gilbert MerLio, Alain Ruiz, Le National-socialisme: une révolution?, Toulouse, 1997, p. 226.

112 Hettler/Grasser, Études haguenoviennes (note 83), p. 169.

113 TO, André Claus, 9 septembre 2002.

114 Kriegswinterhilfswerk.

115 R. Bour, Un Lorrain dans la Kriegsmarine (note 37), p. 34.

116 Présente en Alsace, voir ABBR, 402 D 31, Dossiers d'interdiction de séjour (F. H. à Schiltigheim).

117 Cette composante attire forcément de nombreux jeunes, comme A. F. (né à Obernai (Bas-Rhin) en 1925), qui devient Kamaradschaftsführer uniquement, affirme-t-il, par passion pour l'aviation (ADBR, 544 D 237, Liste d'éventuels justiciables. Comité départemental d'épuration, 1945) ou H. M., qui rejoint la section de Schiltigheim (id., 402 D 18, PV de la Sûreté nationale, 27 septembre 1945).

118 G. Humbert, « Les grandes lignes de la politique allemande » (note 100), p. 193.

119 L. Kettenacker, «La politique de nazification en Alsace» (note 89), p. 85.

120 Angéline Winé, Les Habitants de Dieffenbach-au-Val et la Seconde Guerre mondiale, Master 2, Université de Strasbourg, 2009, p. 40. 
Strasbourg, confection de gants en peau de lapin pour les combattants ${ }^{(121)}$. Au sein des sections l'ambiance est très variable. Tout dépend de l'attitude du responsable local. Les zélateurs du régime se heurtent souvent à une forte résistance passive. Du 17 au 19 juillet 1942 ont lieu à Strasbourg les Jeux de combat d'été de la HJ du Rhin supérieur (Sommer-Kampf-Spiele der oberrheinischen Hitler-Jugend). Derrière un jeune lanceur de poids et sa blonde compagne se profile un soldat arme au pied, ce qui résume bien l'idéal proposé par le régime à la jeunesse alsacienne ${ }^{(122)}$.

Il existe cependant une concurrence entre plusieurs organisations du régime en ce qui concerne les sports, coiffés depuis le 21 décembre 1938 par une autre filiale du parti, la Ligue nationale-socialiste du Reich pour les exercices physiques (Nationalsozialistischer Reichsbund für Leibesübungen - NSRL), qui s'évertue à empêcher les empiétements continuels de la HJ, du DAF, de la SS et de la SA dans ce domaine. Le Gau Oberrhein et le Gau Westmark sont dotés comme les autres d'un Gaubeaufträgter für Leibesübungen (chargé d'affaires pour l'éducation physique) flanqué d'un responsable (Bereichfachwart) pour chaque discipline ${ }^{(123)}$. Le $1^{\text {er }}$ octobre 1940, toute organisation sportive qui n'a pas sollicité son admission au sein du NSRL est interdite ${ }^{(124)}$.

Dissous les 3 septembre et 31 octobre en Alsace et en Moselle ${ }^{(125)}$, les groupements d'avant-guerre, comme L'Avant-garde $d u$ Rhin $^{(126)}$, la Lorraine sportive ${ }^{(127)}$, sont donc réduits à l'impuissance, désormais incapables de remplir leur mission de maintien de l'esprit français et de la foi catholique, qui avait été l'une de leurs principales caractéristiques lors de la première Annexion. Toutefois, la politique des deux Gauleiter n'est pas tout à fait identique: si l'ensemble des activités sportives est absorbé par le NSRL en Alsace, certaines sociétés interdites se reforment en Lorraine sous une dénomination nazie et mènent une existence plus autonome dans le cadre de LandesTurn- und Sportgemeinde Westmark (communauté régionale de gymnastique et de sport Westmark) ${ }^{(128)}$.

\section{Le NSKK, la SA, la SS et les autres organisations paramilitaires}

Parmi les organisations nazies, le Corps motorisé (Nationalsozialistisches Kraftfahrkorps - NSKK), créé en 1931, s’est donné pour but principal la formation de

121 La Mémoire en éveil. Lycée Jean Geiler de Kaysersberg - Strasbourg, Strasbourg, 1998, p. 60.

122 Georges Foessel, Jacques Granier, Alphonse Irjud, La Libération de Strasbourg, Strasbourg, 1994 , p. 20.

123 Dominique CHicAN, «L’Éducation physique en Alsace sous l'occupation allemande (1940-1944). Éléments d'une politique de germanisation? ", in: André RAUCH, Sports et loisirs en Alsace au XX siècle, Paris-Strasbourg, 1994, p. 49.

124 L. Kettenacker, «La politique de nazification en Alsace " (note 89), p. 20.

125 E. SCHAEFFER, L'Alsace et la Lorraine (note 31), p. 94-95.

126 Voir Serge SAint-Michel, Pierre Wachs, L'Avant-garde du Rhin: 90 ans d'histoire, Lingolsheim, 1988; Jean-Marie Le Minor, L'Avant-garde du Rhin, Saint-Cyr-sur-Loire, 2007.

127 Voir Caroline Decleir, La «Lorraine sportive »: une association francophile en Terre d'Empire (19081912), Maîtrise, Strasbourg, 1999.

128 Geneviève Humbert, «Les Activités sportives de la Hitlerjugend dans les départements français occupés du Bas-Rhin, du Haut-Rhin et de la Moselle, 1940-1945 », in: Alfred WAHL, Des jeux et des sports, Metz, 1986, p. 187. 
conducteurs de véhicules pour la Wehrmacht. Désireux de s'assurer le monopole de l'organisation des sports mécaniques, il entend en développer le goût, notamment par le biais de sa revue Der NSKK-Mann ${ }^{(129)}$. Ce corps participe de l'image moderne que veut se donner un régime dont le Führer ne manque jamais de rappeler son amour de la science et de la technique. Dans les publications qui concernent l'Alsace-Moselle pendant la Seconde Guerre mondiale le NSKK est en général présenté comme l'association la moins nazie, une sorte d'Automobile club (d'après $\mathrm{M}^{\mathrm{gr}}$ Pierre Bockel (19141995), aumônier de la brigade Alsace-Lorraine, il « réunit surtout ceux qui redoutent de s'engager dans une section purement politique " ${ }^{(130)}$ et qui veulent passer leur permis de conduire à une époque où rares sont ceux qui ont les moyens de posséder une automobile; il permet aussi d'obtenir de l'essence). Parce que « chacun devait être dans un organisme politique », Alfred Bapst entre au NSKK sur les conseils d'un ami («Si tu rentres là-dedans, ils te foutent la paix »). Content de conduire un camion, il n'assiste aux réunions qu' « une fois de temps en temps, au bistro " ${ }^{(131)}$.

La politique n'est pourtant pas absente au NSKK, dont chaque membre, affirme son chef, Adolf Hühnlein (1881-1942), « est toujours, et en première ligne, un soldat politique d'Adolf Hitler " ${ }^{(132)}$. On y trouve aussi des fanatiques, tel le Scharführer (chef de troupe) de la section de Pfaffenhoffen (Bas-Rhin), membre du parti, qui fait preuve d'une énergie débordante et assure la formation à la conduite des motos des candidats issus de la $\mathrm{HJ}^{(133)}$. En fait, précisait $\mathrm{M}^{\mathrm{gr}}$ Bockel dès 1943, sous le couvert du Témoignage chrétien, l'organisme "dissimule une sorte de préparation militaire aux formations motorisées " ${ }^{(134)}$, et il faut évidemment inculquer dès que possible aux futurs soldats le sens du devoir et de la discipline.

À l'autre extrémité de la « carrière ", les anciens combattants comptent eux aussi parmi les cibles privilégiées du régime. La Ligue nationale-socialiste des soldats du Reich (Nationalsozialistischer Reichskriegerbund - NSRKB), implantée en Alsace en septembre 1940 par le Reichskriegerführer lui-même, le général Reinhard, organise une première grande manifestation à Strasbourg le 20 octobre 1940 avec pour titre: "Les courageux soldats de la Première Guerre mondiale » ${ }^{(135)}$. La Ligue accueille les anciens combattants qui en font spontanément la demande - il s'en trouve - et ceux qui se sentent obligés de le faire. L'appartenance active au NSRKB est l'un des chemins du ralliement qu'empruntent certains anciens de l'armée du Kaiser, peu considérés à l'époque française. Désormais les souffrances endurées pendant la guerre de 1914-1918 sont

129 "National Socialist Motor Korps», in: Bedürftig/Zentner (dir), The Encyclopedia of the Third Reich (note 80), p. 634-635.

130 Pierre Bockel, Alsace et Lorraine terres françaises, Strasbourg, 1975, p. 67.

131 TO, Alfred Bapst, 21 avril 2008.

132 Hans-Helmuth Krenzlin, Das NSKK. Wesen, Aufgaben und Aufbau des Nationalsozialistisches Kraftfahrkorps, dargestellt an einem Ubriß seiner geschichtlichen Entwicklung, Berlin, 1939, p. 5.

133 À l'occasion d'un cours il a des mots avec un jeune homme qu'il traite de Franzosenkopf en ajoutant: « Si je voulais, je pourrais vous mettre à Schirmeck dans les vingt-quatre heures. » En toute circonstance ce personnage "professe une grande confiance dans le Führer » (ADBR, 1366 D 53, Fiches des collaborateurs par commune, G. W., fabricant de chaussures né en 1906, condamné le 24 octobre 1945 à dix ans d'indignité nationale).

134 P. Bockel, Alsace et Lorraine (note 130), p. 65.

135 E. RiEDWEG, Strasbourg (note 21), p. 59. 
pleinement reconnues et même magnifiées. Pour quelques-uns des nombreux survivants l'heure est venue de prendre leur revanche, comme c'est le cas du notaire Daesslé, de Sierentz, ainsi dénoncé le 22 janvier 1946 dans le journal socialiste Le Républicain du Haut-Rhin par Émile Ehrhart (alias « le Maquisard ») : Un nazi 150 \%, signataire du Heimatbundmanifest ${ }^{(136)}$, grand matador de l'autonomisme avant la guerre, hitlérien à toute épreuve dès l'occupation allemande, Ortspropagandaleiter, président du Reichskriegerbund, organisateur d'innombrables réunions de propagande pour le nationalsocialisme, annonciateur du III $^{\mathrm{e}}$ Reich millénaire, membre de la $5^{\mathrm{e}}$ colonne, traître notoire, ayant arboré le premier jour de l'occupation la croix de fer de $1^{\text {re }}$ classe, s'étant pavané dans tous les uniformes bruns et jaunes possibles et imaginables " ${ }^{(137)}$.

Le SA-Gruppe Oberrhein, commandé par le Gruppenführer Leopold Damian, est installé Taulerring à Strasbourg ${ }^{(138)}$. Ainsi que les autres organisations, la SA comprend des membres alsaciens et mosellans inscrits sous la contrainte ou sous la pression de leur entourage (" pour avoir la paix », " pour ne pas perdre mon travail »), mais aussi un certain nombre de militants véritables, dont fait assurément partie Albert Bandel, personnage qui, précise sa fiche d'épuration, a « joué un assez triste rôle pendant les années d'occupation " ${ }^{(139)}$. Vêtu d'un uniforme, le SA-Mann symbolise l'emprise politique et paramilitaire nazie sur la société alsacienne et lorraine. Certains de ces responsables de la SA viennent d'horizons a priori inattendus, comme le chef local à Hoerdt (Bas-Rhin), un cheminot anciennement membre du parti communiste ${ }^{(140)}$. Mais la France a bien Jacques Doriot.

Un autre cas remarquable est celui d'une famille nazie domiciliée à Wiwersheim (Bas-Rhin). Le père, J. M., chef des cultivateurs à Neugartheim (Bas-Rhin), exerce sur eux toutes sortes de pressions pour les amener à livrer les denrées imposées et il manifeste en toute occasion son aversion pour la France ${ }^{(141)}$. Ses trois fils se distinguent dans le même sens, tout spécialement l'aîné, pourtant ancien engagé volontaire dans la marine française ${ }^{(142)}$, Sturmführer et propagandiste de la SA, rendu responsable de l'internement de trois jeunes gens à Schirmeck ${ }^{(143)}$; il se rend si odieux qu'après la Libération un voisin lui conseille vivement de ne plus se montrer au village, "car il se fera lyncher par toute la population ${ }^{(144)}$.

136 Publié en juin 1926, ce texte est la profession de foi du mouvement autonomiste dirigé par Eugène Ricklin.

137 ADBR, 544 D 254, RG de Mulhouse, note d'information, 22 janvier 1946.

138 Il comprend pour l'Alsace les SA-Standarten 132 et 43 à Strasbourg, 99 à Saverne, 137 à Haguenau, J 8 à Sélestat, 171 à Colmar et 112 à Mulhouse (AMS, 207 MW 11, Document daté du 19 janvier 1942 donnant la liste des formations SA et les coordonnées de leurs dirigeants).

139 Né à Monswiller en 1906, ce serrurier est condamné à trois ans de prison par arrêt de la cour de Justice en date du 4 septembre 1945 (ADBR, 1366 W 13, Commissaire principal de Saverne au Sous-préfet, 13 avril 1948).

140 Id., 402 D 28, Dossiers d'interdiction de séjour (C. W.).

141 ADBR, 402 D 17, Secrétaire général chargé de l'administration de l'arrondissement de StrasbourgCampagne, 3 mars 1945.

142 ADBR, 402 D 17, PV de la gendarmerie de Truchtersheim, 8 août 1945.

143 Id., 28 mai 1945.

144 Id., PV de la gendarmerie de Truchtersheim, 28 mai 1945. 
En Moselle est implantée le $1^{\text {er }}$ octobre 1940 la SA-Brigade 150, qui se divise en cinq Standarten d'infanterie - à Metz, Saint-Avold, Thionville, Sarreguemines et Sarrebourg -, un Standarte de cavalerie à Metz et un SA-Marinesturmbann (groupe d'assaut de marine $)^{(145)}$, correspondant à un bataillon. Comme la HJ, la SA comprend en effet des sections spécialisées: musique ${ }^{(146)}$, activités nautiques ou même militaires (Panzerkorps Feldherrnhalle) ${ }^{(147)}$.

Au sein de la SS, édifice à plusieurs étages, la SS générale (Allgemeine SS) regroupe des personnes qui exercent une profession civile ${ }^{(148)}$. Leur service, effectué les samedis après-midi et les dimanches, consiste en des tournées de propagande en faveur de l'engagement dans les Waffen SS, la participation à des réunions politiques, à des activités physiques et à des entraînements militaires ${ }^{(149)}$. En Alsace et en Moselle, comme ailleurs, les clubs comptent des adhérents aux motivations diverses, certains uniquement intéressés par le sport, comme le montre l'exemple de P. F., chef cantonnier à Strasbourg, qui « lors de la fusion de ladite formation avec l'Allgemeine SS s'en est désintéressé complètement ${ }^{(150)}$. D’autres sont visiblement là autant, voire davantage, par sympathie pour l'idéologie que par passion pour la discipline qu'ils pratiquent. C'est aussi un lieu de sociabilité pour ceux qui songent à faire carrière dans un domaine quelconque.

\section{Le RAD et le KHD}

Le volontariat dans le Service national du Travail ou Reichsarbeitsdienst (RAD) est possible pour les Alsaciens à partir du 5 février $1941^{(151)}$ et des appels en ce sens sont lancés dans les Straßburger Neueste Nachrichten des 20 et 28 février ${ }^{(152)}$. Devant le peu de succès rencontré par cette campagne, le RAD est rendu obligatoire le 23 avril en Moselle et le 8 mai en Alsace ${ }^{(153)}$ (où il concerne les garçons des classes 1920 à 1927 et les filles des classes 1923 à 1926). « Les parents alsaciens confieront de grand cœur leurs fils et leurs filles à cette communauté de Travail, précise le décret du Gauleiter, car au RAD la jeune recrue, qu'elle sorte de l'enseignement secondaire, du bureau ou de la fabrique comprendra le national-socialisme par la pratique " ${ }^{(154)}$. Après les premiers conseils de révision (Musterungen) en août, le premier contingent est incorporé en octobre ${ }^{(155)}$.

\section{ADM, 2 W 92.}

146 ADBR, 402 D 28, Dossiers d'interdiction de séjour (Albert Schirmann à Bischheim) et 402 D 31, Dossiers d'interdiction de séjour (Léon Arnold, également à Bischheim).

147 Site Axis History Factbook.

148 Marlis G. Steinert, « L’Ordre noir de la SS », in: François BédArida (dir.), L'Allemagne de Hitler 1933-1945, Paris, 1991, p. 101.

149 Henri Mounine, Cernay 40-45. Le SS-Ausbildungslager de Sennheim, Ostwald, 1999, p. 61.

150 ADBR, 544 D 241, PV du comité départemental d'épuration, 29 mai 1946 (personne non sanctionnée).

151 AMS, 21 MW 197, Ordonnance du ministre de l’Intérieur (Reichsminister des Innern), 5 février 1941.

152 Id., Coupures de presse.

153 Id., Ordonnance du ministre de l’Intérieur, 8 mai 1941.

154 Paul PilAnt, «La Jeunesse alsacienne enrégimentée par les nazis », L’Information historique, $14^{\mathrm{e}}$ année, $\mathrm{n}^{\circ} 1$, janvier-février 1952, p. 101.

155 Eugène RiEdwEG, « Le RAD, un service pas très civil », Saisons d'Alsace (note 73), p. 96. 
Les jeunes filles requises pour le $\mathrm{RAD}^{(156)}$ sont détachées comme assistantes ménagères, comme employées dans des commerces (boulangeries, hôtels) ou pour les travaux agricoles $^{(157)}$. Elles sont confrontées au même dilemme que les garçons, comme l'explique une Messine: "Si on refusait, c'est toute la famille qui était déportée " ${ }^{(158)}$. Le conseil de révision se révèle une épreuve particulièrement pénible. " Notre première humiliation fut la visite médicale, avant le RAD, raconte Simone Vilm, convoquée avec sa sœur. Nous étions toutes nues et l'on nous donna un verre pour l'examen d'urine. Devant les hommes, c'était dur ${ }^{(159)}$. D'autres se posent moins de questions. Incorporée de force le 12 juillet 1943, Simone Gargoët avoue avec franchise: « J'étais l'aînée et tout le boulot retombait sur moi. Ma seconde mère ${ }^{(160)}$ n'était pas toujours très gentille avec nous. J'étais donc bien contente de quitter le giron familial et je me suis dit: “J’arriverai bien à me débrouiller, peu importe où je vais" »(161).

Quatre cents jeunes filles de Colmar et sept cents de Strasbourg partent pour l'Allemagne dès novembre 1941. Pour beaucoup de recrues féminines, le RAD est suivi par une nouvelle période dans le cadre du Kriegshilfsdienst (KHD). Ce service auxiliaire de guerre pour la jeunesse féminine est instauré par décret du 29 juillet 1941. En principe limité au territoire du Reich, il concerne en fait de nombreux théâtres d'opérations ${ }^{(162)}$. Il est introduit en Alsace le $1^{\text {er }}$ octobre 1941, mais ce n'est qu'un an plus tard que les premières Alsaciennes y sont assujetties ${ }^{(163)}$. Là encore la mesure suit l'échec d'un appel au volontariat ${ }^{(164)}$. Le 6 juillet 1942, l'enrôlement dans le KHD est également décrété en Moselle (3000 personnes furent finalement concernées) ${ }^{(165)}$. En sont généralement dispensées les filles d'agriculteurs, les étudiantes, les femmes mariées. Dans de nombreuses communes rurales, comme à Oberentzen (Haut-Rhin), aucune jeune fille n'est concernée ${ }^{(166)}$. Posséder un emploi est une bonne garantie. "Ma sœur travaillait à l'Hôpital civil (de Strasbourg) à l'époque, en radiologie centrale, comme secrétaire, elle y est restée ", confirme André Claus ${ }^{(167)}$.

Au total, le nombre des Alsaciens et des Lorrains mosellans incorporés de force dans le RAD est estimé à 70000 personnes, dont 15000 jeunes filles ${ }^{(168)}$. Le RAD

156 Voir Laure BAlzANO-DupuiCH, Les Femmes alsaciennes incorporées de force dans les organisations de l'Allemagne nazie, 1941 à 1945, Maîtrise, Strasbourg II, 2003; Madeleine AnstetT, Les Malgré-elles, Master 2, Strasbourg, 2013; Joseph EhrHARD, «Les Malgré-elles », Annuaire de la Société d'histoire et d'archéologie du Ried Nord, 2004: L'Incorporation de force. Témoignages de Malgré-nous, p. 271-276.

157 Bedürftig/Zentner (dir.), The Encyclopedia of the Third Reich (note 80), p. 775.

158 Dernières Nouvelles d'Alsace (DNA) - Version femme, 13 avril 2001.

159 Gandebeuf/Thiriet, La Parole retrouvée (note 10), p. 167.

160 Son père était remarié.

161 TO, Simone Gargoët-Nevels, 25 novembre 2002.

162 ADM, 1928 W 21, Dossier personnel de F. M., née en 1923 à Béning-lès-Saint-Avold (Moselle). DIACLCA.

163 L. Balzano-Dupuich, Les Femmes alsaciennes incorporées de force (note 156), p. 80.

164 E. Riedweg, Les Malgré-nous (note 84), p. 148.

165 ONAC de Moselle, L'Incorporation de force en Moselle. Les Malgré-nous, une jeunesse sacrifiée, Metz, 2005, s.p.

166 ADHR, 1441 W 3, Enquête... Commune d'Oberentzen, 23 août 1950.

167 TO, André Claus, 9 septembre 2002.

168 DNA, 11 avril 1999. 
s'inscrit dans un cadre global d'assimilation, que résume assez bien en peu de mots un Alsacien ensuite incorporé de force: "On nous a dressés » ${ }^{(169)}$. Pour la première fois, les nazis exigent des annexés de fait une reconnaissance explicite de leur appartenance à l'Allemagne en les contraignant à prêter serment à Hitler. La prochaine étape, beaucoup le craignent dès 1941, est l'instauration de la conscription car si le service paramilitaire est une chose, la solidarité du front, la mort affrontée côte à côte en est une autre. «Ce n'est que lorsque l'Alsace tremblera, comme tous les autres Gaue du Reich, pour ses pères et ses fils, que la guerre pourra servir de catalyseur d'où sortira en quelque sorte, telle une nouvelle combinaison chimique, la communauté du peuple des Alsaciens et des Allemands » ${ }^{(170)}$.

\section{Conclusion}

À partir de juin 1940, l'Alsace et la Moselle changent de sphère culturelle et idéologique. Le retour dans le giron allemand signifie aussi le retour dans la guerre. Alors que le reste de la France est encore en grande partie épargné par ses conséquences les plus tragiques (bombardements, réquisitions, répression, déportation) - même s'il connaît la pénurie -, alors que la moitié du pays bénéficie encore du statut de "zone libre ", les Alsaciens et les Lorrains mosellans sont soumis à une propagande de tous les instants et à des pressions sans commune mesure avec ce qui se déroule ailleurs dans le pays. Une minorité y répond favorablement, avec plus ou moins d'enthousiasme, tandis que la majorité de la population fait preuve d'attentisme en se contentant des apparences. C'est ainsi que Philippe Mischler, de Hoerdt (Bas-Rhin), « manifestait des sentiments différents selon qu'il se trouvait en présence de personnes francophiles ou germanophiles " ${ }^{(171)}$. Il n'est sans doute pas le seul, et beaucoup d'autres jugent encore plus prudent (et d'ailleurs plus digne) de se taire et de n'exprimer aucun avis politique.

Les résultats de la «mise au pas » n'ont pas encore fait l'objet d'une étude suffisamment approfondie. Cette contribution n'est qu'un rapport d'étape. Il serait nécessaire, notamment, d'établir des statistiques concernant le nombre d'adhérents aux différentes formations nazies. Un chercheur de l'Université de Mannheim affirme, par exemple, que les entrées dans la NSDAP d'Alsaciens « de souche » (encore faut-il s'entendre sur une définition de ce terme) seraient bien plus nombreuses que ce que l'on laissait entendre jusque-là ${ }^{(172)}$. La multiplication des monographies sur le sujet permettra sans doute un jour de se faire une idée plus précise de la question. Toutefois, de nombreuses zones d'ombre - celles inhérentes à l'âme humaine - subsisteront toujours. On ne peut en effet se fier aux chiffres officiels lorsqu'il s'agit d'un État totalitaire, dans lequel des mots tels que «Freiwillige" (volontaire) ont également une valeur toute relative. Par ailleurs, les fiches d'épuration, si elles contiennent une foule d'informations intéressantes, ne constituent pas non plus une source infaillible. Dans

169 TO, Lucien Hauler, Dessenheim, 12 octobre 2004.

170 L. Kettenacker, «La politique de nazification en Alsace» (note 89), p. 90.

171 ADBR, 402 D 18, Rapport de la section de gendarmerie de Strasbourg-Campagne, 28 mai 1945.

172 Markus Enzenauer, «Deutsches Elsass, kehre heim », in: Konrad Krimm (dir.), NS-Kulturpolitik und Gesellschaft am Oberrhein 1940-1945, Stuttgart, 2013, p. 15-79. 
tous les cas il faudra tenir compte du fait que l'Alsace et la Moselle sont les seules régions de France à avoir fait l'expérience du totalitarisme (car on ne peut comparer une occupation militaire à une annexion de fait). Cela change tout dans la manière d'appréhender les sources.

\section{Résumé}

Au cours de la Seconde Guerre mondiale, l'Alsace et la Moselle ont été annexées de fait, en juillet 1940, par le III Reich. Ce sont les seules régions de France à avoir fait l'expérience du totalitarisme (car on ne peut comparer une occupation militaire à une annexion de fait). Leur "mise au pas " (Gleichschaltung) passe par une phase de défrancisation (retour à un état de choses naturel interrompu en 1918, selon l'idéologie nouvelle), de décléricalisation (volonté d'arracher l'âme de la population, et surtout de la jeunesse, aux très influentes Églises), de germanisation (lutte contre la langue et la culture françaises) et enfin de nazification (avec l'importation de toutes les organisations liées au parti nazi). Les habitants sont soumis à une propagande de tous les instants. Bien que toutes les sources n'aient pas encore été exploitées et que cette exploitation soit parfois malaisée, on peut affirmer que seule une minorité y répond favorablement - mais on manque encore de statistiques définitives -, avec plus ou moins d'enthousiasme, tandis que la majorité de la population fait preuve d'attentisme et se contente des apparences.

\section{Zusammenfassung}

Während des 2. Weltkrieges wurden das Elsass und das Département Moselle im Juli 1940 durch das III. Reich de facto annektiert. Ihre Erfahrung des Totalitarismus ist einzig in Frankreich, denn eine militärische Besatzung ist nicht vergleichbar mit einer de facto Annexion. Die Gleichschaltung der annektierten Region war gekennzeichnet durch eine Phase der "Entwelschung" (zum Zweck der Rückkehr zu einem im Sinne der neuen Ideologie natürlichen, 1918 unterbrochenen Zustand), der Entklerikalisierung (mit dem Ziel, die Bevölkerung, vor allem die Jugend, den einflussreichen Kirchen zu entfremden), der Germanisierung (Bekämpfung der französischen Sprache und Kultur), und der Nazifizierung (durch den Import aller NS-Organisationen). Die Bevölkerung war einer ständigen Propaganda ausgesetzt. Obwohl alle Quellen noch nicht ausgewertet sind und trotz fehlender Statistiken kann behauptet werden, dass nur eine Minderheit sich mehr oder weniger einnehmen ließ, während die Mehrheit sich abwartend verhielt. 